\title{
A general purpose Fortran 90 electronic structure program for conjugated systems using Pariser-Parr-Pople model
}

\author{
Priya Sony ${ }^{1}$, Alok Shukla ${ }^{2}$ \\ Department of Physics, Indian Institute of Technology, Bombay, Powai, Mumbai \\ 4000\%6, INDIA
}

\begin{abstract}
Pariser-Parr-Pople (P-P-P) model Hamiltonian has been used extensively over the years to perform calculations of electronic structure and optical properties of $\pi$ conjugated systems successfully. In spite of tremendous successes of $a b$ initio theory of electronic structure of large systems, the P-P-P model continues to be a popular one because of a recent resurgence in interest in the physics of $\pi$-conjugated polymers, fullerenes and other carbon based materials. In this paper, we describe a Fortran 90 computer program developed by us, which uses P-P-P model Hamiltonian to not only solve Hartree-Fock (HF) equation for closed- and open-shell systems, but also for performing correlation calculations at the level of single configuration interactions (SCI) for molecular systems. Moreover, the code is capable of computing linear optical absorption spectrum at various levels, such as, tight binding (TB) Hückel model, HF, SCI, and also of calculating the band structure using the Hückel model. The code also allows the user to solve the HF equation in the presence of finite external electric field, thus, permitting calculations of quantities such as static polarizabilities and electro-absorption spectra. Additionally, it can perform transformation of P-P-P model Hamiltonian from the atomic orbital (AO) representation (also called site representation) to the molecular orbital (MO) one, so that the transformed matrix elements can be used for high level post-HF calculations, such as, full CI (FCI), quadruple CI (QCI), and multi-reference singles-doubles CI (MRSDCI). We demonstrate the capabilities of our code by performing calculations of various properties on conjugated systems such as trans-polyacetylene ( $t$-PA), poly-para-phenylene (PPP), poly-para-phenylene-vinylene (PPV), oligo-acenes, and graphene nanodisks.
\end{abstract}

Key words: Hartree-Fock method, self-consistent field approach PPP model Hamiltonian, Molecular orbitals PACS: 31.15.xr, 31.15.Ne, 31.15.bu, 31.15.-p 
1 e-mail: psony@phy.iitb.ac.in

2 Author to whom all the correspondence should be addressed. e-mail: shukla@phy.iitb.ac.in 


\section{Program Summary}

Title of program: ppp.x

Catalogue Identifier:

Program summary URL:

Program obtainable from: CPC Program Library, Queen's University of Belfast, N. Ireland

Distribution format: tar.gz

Computers : PC's/Linux

Linux Distribution: Code was developed and tested on various recent versions of Fedora including Fedora 11 (kernel version 2.6.29.4-167)

Programming language used: Fortran 90

Compilers used: Program has been tested with Intel Fortran Compiler (noncommercial version 11.1) and gfortran compiler (gcc version 4.4.0) with optimization option -O.

Libraries needed: This program needs to link with LAPACK/BLAS libraries compiled with the same compiler as the program. For the Intel Fortran Compiler we used the ACML library version 4.3.0, while for gfortran compiler we used the libraries supplied with the Fedora distribution.

Number of bytes in distributed program, including test data, etc.: size of the tar file ...... bytes

Number of lines in distributed program, including test data, etc.: lines in the tar file

Card punching code: ASCII

Nature of physical problem: The problem of interest at hand is the electronic structure of $\pi$-conjugated systems. For such systems, the effective $\pi$-electron P-P-P semi-empirical model Hamiltonian proposed by Pariser, Parr, and Pople offers an attractive alternative as compared to the ab initio approaches. The present program can solve the HF equations for both open- and closed-shell systems within the P-P-P model. Moreover, it can also include electron correlation effects at the singles CI level. Along with the wave functions and energies, various properties such as linear absorption spectra can also be computed.

Method of Solution: The single-particle HF orbitals of a $\pi$-conjugated system are expressed as linear combinations of the $p_{z}$-orbitals of individual atoms (assuming that the system is in the $x y$-plane). Then using the hopping and Coulomb parameters prescribed for the P-P-P method, the HF integro-differential equations are transformed into a matrix eigenvalue problem. Thereby, its solutions are obtained in a self-consistent manner, using the iterative diagonalizing

1 e-mail: psony@phy.iitb.ac.in

2 Author to whom all the correspondence should be addressed. e-mail: shukla@phy.iitb.ac.in 
technique. The HF orbitals thus obtained can be used to perform a variety of calculations such as the SCI, linear optical absorption spectrum, polarizabilty, electro-absorption spectrum, etc.

Running Time: The examples provided each only take a few seconds to run. For a large molecule or a polymer, however, the run time may be a few minutes.

Unusual features of the program: None

\section{Introduction}

The studies of electronic structure and optical properties of $\pi$-conjugated systems have fascinated physicists and chemists alike for a long time[1,2], because of their possible applications in opto-electronic devices such as OLEDs, OFETs, organic lasers, solar cells, etc[3]. Ever since graphene and its nanostructures such as graphene nano-ribbons, nano-disks, etc. have been synthesized[4], interest in the study of $\pi$-conjugated systems has multiplied many folds[5].

In the 1950's, Pariser, Parr, and Pople proposed a simple model which incorporates the essential physics of correlated conjugated systems in a very elegant manner[6]. In this model only $\pi$-electrons are considered explicitly, while the effect of $\sigma$-electrons is included in an implicit manner in terms of various parameters. Moreover, long range electron-electron interactions are taken into account by means of suitable Coulomb parameters. Thus, one reduces the number of degrees of freedom involved in the underlying Schrödinger equation considerably. Because of the lack of large scale computational facilities during the 1950's such an approach was unavoidable even for relatively small molecules such as benzene. However, the remarkable fact is that in spite of so many approximations involved, P-P-P model based calculations were extremely successful in describing the electronic structure, in general, and the optical properties of such systems, in particular[2].

Now, the question arises that in the present times, when computational power has grown by orders of magnitude as compared to those in 1950's, why should one be still interested in using the P-P-P model, particularly, when so many sophisticated $a b$ initio electronic structure programs are available. In our opinion there are two main reasons behind the continued use of the P-P-P model at present: (a) One gets to understand the electronic and optical properties of such systems within a rather simplified picture, in terms of the dynamics of the $\pi$-electrons, (b) Ever since the revival of interest in the $\pi$-conjugated polymers because of their possible applications in opto-electronics, the size of the systems being investigated these days is becoming quite large consisting of hundreds of atoms, pendant side groups, and terminal functionalities. Therefore, simultaneous high quality $a b$ initio description of both the ground 
and the excited states of such systems (needed to describe their linear and nonlinear optical properties) is virtually impossible. While, the P-P-P model, with its reduced degrees of freedom, allows one to compute low-lying excited states of rather large systems using the present computational capacities.

Indeed a large number of calculations on various conjugated systems have been performed in last several years using the P-P-P model Hamiltonian with a great deal of success[1,7,8,9,10,11,12,13]. In our own group and with our collaborators, we have performed several calculations on systems as diverse as phenyl disubstituted polyacetylenes (PDPAs)[14,15,16,17,18,19,20], PPVs[21,22,23], and oligo-acenes[24,25,26]. In all the afore-mentioned works the HF calculations and the transformation of the Hamiltonian from the AO to the MO representation were performed using a Fortran 77 code, developed earlier in our group[27]. However, the code in question lacked the capability of performing unrestricted Hartree-Fock (UHF) calculations, and thus, was limited only to closed-shell systems. Moreover, Fortran 77 language did not allow dynamic memory allocation, resulting in a dependence on compile-time dimensional parameters. Therefore, we have rewritten the code in a modern programming language, namely, Fortran 90, which allows it to utilize dynamic memory allocation, thereby freeing it from various array limits, and resultant artificial restrictions on the size of the molecules. Thus our program can be used on a given computer until all its available memory is exhausted. The present computer program can perform restricted Hartree-Fock (RHF) calculations on closed-shell systems, as well as unrestricted-Hartree-Fock (UHF) calculations on open-shell systems. Moreover, like the earlier version of the code[27], it can also carry out correlation calculations at the level of singles configuration interactions (SCI) for molecular systems. Additionally, the code is capable of computing optical spectrum at various levels, such as, TB, HF, and SCI, and also of calculating the band structure using Hückel model. The program also allows user to solve HF equation in the presence of an external electric field, thus, permitting calculation of dielectric polarizabilities and electro-absorption spectrum of conjugated molecules. Additionally, it can perform transformation of P-P-P model Hamiltonian from the AO representation (site representation) to the $\mathrm{MO}$ one, so that the results can be used for high level post HF correlated calculations, such as, full CI (FCI), quadruple CI (QCI), and multi-reference singles-doubles CI (MRSDCI). The other features of the code include the charge density analysis and the molecular orbital analysis. Apart from describing the computer program, we also present several of its applications which include various examples for $t$-PA, PPP, PPV, acenes, and graphene nanodisks.

The remainder of the paper is organized as follows. In section 2 we briefly review the theory associated with the P-P-P model Hamiltonian. Next, in section 3 we discuss the general structure of our computer program, and also describe its constituent subroutines. In section 4 we briefly describe how to 
install the program on a given computer system, and to prepare the input files. Results of various example calculations using our program are presented and discussed in section 5. Finally, in section 6, we present our conclusions, as well as discuss possible future directions.

\section{Theory}

In this section we briefly review the theory associated with the P-P-P model Hamiltonian and provide the necessary HF equations within the basis set approach.

\subsection{Hamiltonian}

The P-P-P Hamiltonian can be written as

$$
\begin{aligned}
H_{P P P}= & \sum_{i, \sigma} \epsilon_{i} c_{i \sigma}^{\dagger} c_{i \sigma}+\sum_{\langle i j\rangle, \sigma} t_{i j}\left(c_{i \sigma}^{\dagger} c_{j \sigma}+c_{j \sigma}^{\dagger} c_{i \sigma}\right)+U \sum_{i} n_{i \uparrow} n_{i \downarrow} \\
& +\sum_{i<j} V_{i j}\left(n_{i}-1\right)\left(n_{j}-1\right)
\end{aligned}
$$

where $\epsilon_{i}$ represents the site energy associated with the $i$ th atom, $\langle i j\rangle$ implies nearest neighbors, $c_{i \sigma}^{\dagger}$ creates an electron of spin $\sigma$ on the $p_{z}$ orbital of carbon atom $i$ (assuming that the system is lying in the $x y$-plane), $n_{i \sigma}=c_{i \sigma}^{\dagger} c_{i \sigma}$ is the number of electrons with spin $\sigma$, and $n_{i}=\Sigma_{\sigma} n_{i \sigma}$ is the total number of electrons on atom $i$. The parameters $U$ and $V_{i j}$ are the on-site and longrange Coulomb interactions, respectively, while $t_{i j}$ is the nearest-neighbor oneelectron hopping matrix element.

The particular forms of the parameters $U$ and $V_{i j}$ determine the specific variants of the P-P-P model Hamiltonian[7]. For $U \neq 0$, and $V_{i j}=0$, the model becomes the Hubbard Hamiltonian, while for $U \neq 0$, and $V_{1} \neq 0$, but all $V_{i j}=0$, we obtain the extended Hubbard model. Choosing for the long-range $V_{i j}$ the form

$$
V_{i j}=\frac{U}{\left[1+\left(\frac{R_{i j}}{r_{0}}\right)^{2}\right]^{1 / 2}},
$$

with $U$ and $r_{0}$ fitted to data gives the Ohno variant[28] of the P-P-P model, 
whereas taking

$$
V_{i j}=\frac{U}{\left[1+\left(\frac{R_{i j}}{r_{0}}\right)\right]},
$$

gives the Mataga-Nishimoto variant[29]. In exponential variant[30], $V_{i j}$ takes the following form

$$
V_{i j}=U \exp \left(-\frac{R_{i j}}{r_{0}}\right)
$$

In above Eqs.(2, 3, 4), $R_{i j}=\left|\mathbf{r}_{i}-\mathbf{r}_{j}\right|$ is the distance between sites $i$ and $j$ in $\AA$, while $r_{0}$ is some parameter in the same units.

In this work, we report calculations based upon the Ohno parametrization of the P-P-P model mentioned above (cf. Eq.2). Moreover, to account for the inter-chain screening effects we use a slightly modified form introduced by Chandross and Mazumdar[31],

$$
V_{i j}=U / \kappa_{i j}\left(1+0.6117 R_{i j}^{2}\right)^{1 / 2}
$$

where $\kappa_{i j}$ depicts the dielectric constant of the system which can simulate the effects of screening and $R_{i j}$ is defined above. In various calculations performed on phenylene-based conjugated polymers including PDPAs[14,15,16,17,18,19,22,23], it was noticed that "screened parameters" with $U=8.0 \mathrm{eV}$ and $\kappa_{i i}=1.0$, and $\kappa_{i j}=2.0$, otherwise, proposed by Chandross and Mazumdar[31], lead to much better agreement with the experiments, as compared to the "standard parameters" with $U=11.13 \mathrm{eV}$ and $\kappa_{i, j}=1.0$, proposed originally by Ohno. In the present code we provide the user with the freedom to choose these "standard", "screened", or any other user defined parameters for the Coulomb interactions.

In order to calculate static polarizabilities, one can solve the HF equations in the presence of an external static electric field. Thus, we have modified Eq.(1) under the electric dipole approximation by introducing the corresponding term containing the uniform electric field $\mathbf{E}$. The overall Hamiltonian of the system is then given by

$$
H_{P P P}^{e f i e l d}=H_{P P P}-\boldsymbol{\mu} . \mathbf{E}=H_{P P P}+|e| \mathbf{E} \cdot \mathbf{r},
$$

where $H_{P P P}$ is the unperturbed Hamiltonian that describes the system in the absence of the electric field, $e$ represents the electronic charge, $\boldsymbol{\mu}=-e \mathbf{r}$, is the dipole operator, and $\mathbf{r}$ is the positive operator. 


\subsection{UHF equations}

Now we describe our formulation for the UHF method. The corresponding RHF equations can be easily deduced from them. As per the assumptions of the UHF method, we assume that the $\mu$-th up- and down-spin orbitals are distinct, and are represented (say) as $\psi_{\mu}^{(\alpha)}$ and $\psi_{\mu}^{(\beta)}$, respectively. We assume that these orbitals can be written as a linear combination of a finite-basis set

$$
\psi_{\mu}^{(\alpha)}=\sum_{i} C_{i \mu}^{(\alpha)} \phi_{i}
$$

where $\phi_{i}$ 's represent the $p_{z}$-orbitals in question, and the determination of the unknown coefficients $C_{i \mu}^{(\alpha)}$ amounts to the solution of the UHF equations. It is further assumed that the $\phi_{i}$ orbitals form an orthonormal basis set. In the equation above, we have only stated the expressions for the up-spin orbitals, the case of the down-spin orbitals can be easily deduced. We further assume that the total number of up-/down-spin electrons is $N_{\alpha} / N_{\beta}$, such that $N_{\alpha}+$ $N_{\beta}=N_{e}$. Using the conjecture of Eq. (7) in conjunction with the Hamiltonian above, one obtains the so-called Pople-Nesbet equations

$$
\sum_{j}\left(F_{i j}^{\alpha}-\varepsilon_{\mu}^{\alpha}\right) C_{j \mu}^{(\alpha)}=0
$$

where $\epsilon_{\mu}^{\alpha}$ is the UHF eigenvalue of the $\mu$-th up-spin orbital, $F_{i j}^{\alpha}$ is the Fock matrix for the up-spin electrons defined by the equations

$$
\begin{aligned}
& F_{i j}^{\alpha}=t_{i j}-P_{i j}^{\alpha} V_{i j}, \quad(i \neq j) \\
& F_{i i}^{\alpha}=\epsilon_{i}-\sum_{j \neq i} V_{i j}+\sum_{j} P_{j j} V_{i j}-P_{i i}^{\alpha} V_{i i}, \quad(i=j)
\end{aligned}
$$

where $\epsilon_{i}, t_{i j}$ and $V_{i j}$ are defined above (cf. Eq. 1), and $P_{i j}^{\alpha}$ and $P_{i j}$, are the up-spin and total density matrix elements, respectively, defined as

$$
P_{i j}^{\alpha}=\sum_{\mu=1}^{N_{\alpha}} C_{i \mu}^{(\alpha) *} C_{j \mu}^{(\alpha)},
$$

and

$$
P_{i j}=P_{i j}^{\alpha}+P_{i j}^{\beta}
$$

Once the Fock matrix is constructed, one solves the eigenvalue problem for the up-spin Fock matrix (cf. Eq. 8) as well as the down-spin Fock matrix, using 
the iterative diagonalization technique, to achieve self-consistency. From the

equations given above, it is easy to deduce the expressions for $F_{i j}^{\beta}$, as well as the Fock matrix elements for the RHF case.

\section{Description of the Program}

Our computer code consists of the main program, and various subroutines, all of which have been written in Fortran 90 language. Additionally, the program must link to the LAPACK/BLAS library, whose diagonalization routines are used by our program.

Before we describe various subroutines in detail, it is fruitful to discuss the scaling of the memory requirements, and the cpu time (calculation time), with the system size. If $N$ is the number of sites ( $\pi$ orbitals) in the system, then the memory requirements will roughly scale as $N^{2}$, needed mainly for the storage of the Fock matrix, and its eigenvectors. The cputime, on the other hand, will be dominated by the self-consistency iterations (the so-called "selfconsistent field" (SCF) process), which consists of construction of the Fock matrix, and its diagonalization. The cputime needed for the construction of the Fock matrix scales as $N^{2}$, while the diagonalization time exhibits $N^{3}$ scaling. Thus, we conclude that the dominant scaling of the cputime is $N^{3}$, with respect to the system size. For the open-shell systems, for which the UHF procedure is needed, the memory and cpu time requirements will roughly be twice as compared to a closed shell system of similar size because the UHF procedure involves the construction and diagonalization of two Fock matrices, corresponding to the "up", and the "down" spins.

Several molecules in nature are highly symmetric, and, therefore, in general, it is fruitful to implement the point-group symmetries in electronic-structure codes like the present one. However, our code does not make use of pointgroup symmetries mainly because the computation time associated even for large systems is really insignificant. Therefore, the programming effort required to implement various irreducible representations will simply outweigh the rewards in terms of reduced cpu time. Moreover, the irreducible representations associated with various orbitals and many-particle states can be easily determined by examining the transition dipole moments among them, in conjunction with the dipole-selection rules of various point groups. Next, we briefly describe the main program, and each of the subroutines of our code. 


\subsection{Main program PPP}

This is the main program of our package which reads input data such as which Hamiltonian to use, its parametrization, charge on the system, total number of atoms in the unit cell, and their coordinates. The program also reads in the options to perform various types of calculations such as RHF, UHF, SCI, optics, etc. The option is also provided to perform HF calculations in the presence of an external electric field, which is specified by its Cartesian components in the units of $\mathrm{V} / \AA$. The program can delete the atoms and calculates the entities like total number of sites, electrons and occupied orbitals. It dynamically allocates various arrays, and then calls other subroutines to accomplish the remainder of the calculations. Because of the dynamical array allocation, the user need not worry about various array sizes, as the program will automatically terminate when it exhausts all the available memory on the computer.

\subsection{Subroutine R_ATOM}

This subroutine reads the coordinates of the atoms of a unit cell with respect to its user specified origin. It can also generate coordinates of some important structural units such as a bond, phenyl group (benzene), and even fullerene $\left(\mathrm{C}_{60}\right)$ to facilitate an easy realization of the molecular system under consideration.

\subsection{Subroutine BENPERP}

This routine generates the coordinates of six carbon atoms forming the benzene backbone. The orientation is perpendicular to the conventional orientation. Note that the origin of the benzene skeleton is considered to be the center of the hexagonal ring. Rotations, if desired, are performed keeping the origin

fixed. Positive angles are considered to be anti-clockwise, while the negative angles are treated clockwise. Finally, the center is translated to the location specified by the user. The plane in which benzene locates is taken as a card (XY or YZ or ZX) in the input file.

\subsection{Subroutine BENZEN}

Similar to subroutine BENPERP, this routine also generates the coordinates of six carbon atoms forming the benzene backbone. This routine differ from the 
previous one in a way that it creates benzene ring in the orientation parallel to the conventional orientation. One can also rotate and translate the ring, and can also specify the plane in which benzene ring lies.

\subsection{Subroutine BOND}

This routine generates a bond ( 2 carbon atoms). Center of the bond is taken to be the origin and initially the bond is assumed to be along the x-axis. Rotations, if desired, are performed keeping the origin fixed. Positive angles are considered for anti-clockwise rotation, while the negative angles are treated for clockwise rotation. Finally, the center is translated to the location specified by the user.

\subsection{Subroutine STLINE}

This subroutine also generates a bond, but with the first atom of the bond at the origin. Here, initially the bond is assumed to be along the x-axis. Operations like rotation and translation can also be performed.

\subsection{Subroutine C60_GEN}

This routine, which is originally written by Dharamvir et al.[32], generates the position coordinates of all 60 carbon atoms of the fullerene structure. Inputs are two bond lengths, i.e., single and double bond lengths. This does not have the "standard" orientation, but one with pentagons at top and bottom.

\subsection{Subroutine $R_{-}$SITE}

If the molecule under consideration consists of translationally invariant units, this routine generates the coordinates of all the $\pi$-electron sites in the system from the coordinates of origins of the reference unit cell and the translational vector of the lattice. At present this subroutine is restricted to periodicity only in one dimension, thus, making it useful for polymers and their oligomers. 


\subsection{Subroutine DELATM}

This subroutine deletes the users specified atoms from the list containing all the atomic coordinates.

\subsection{Subroutine SYMSITE}

This routine symmetries the coordinates of the atoms to place the origin of the molecule at the center of mass of the molecule.

\subsection{Subroutine PRINTR}

This routine truncates the position coordinates to seven places to right of the decimal and then prints out the coordinates of all the sites into the output file. In addition, it also creates a file named as 'atomic_coord.xsf', which can be used as input file in XCrySDen[33] program, meant for visualizing the molecular structure of the system under consideration.

\subsection{Subroutine MATEL}

This is the master routine meant for generating the one-electron $\left(t_{i j}\right)$ and two-electron $\left(V_{i j}\right)$ matrix elements, with or without the electric field. This is done based upon the data specified by the user in the input file such as the Hamiltonian under consideration, Coulomb parameters to be used (if any), hopping matrix elements connecting various sites, etc.

\subsection{Subroutine Tij_READB}

This routine is meant for reading the hopping related input for the band structure calculations using the TB model (Hückel model). It reads the unique intracell, as well as intercell, hopping matrix elements, and their connectivities from the input file. Note that an one dimensional tight-binding system with only nearest-neighbor coupling is assumed. Thus, a given cell can only connect to at the most to two neighbors (one to the left and the other to the right). For anything else the code will have to be modified. Also one has to specify the unit cell to which it is connected. 


\subsection{Subroutine READ_EI}

If there any site energies are, this routine reads them. In case of more than one unit cell, the routine also generates rest of the site energies using translational invariance.

\subsection{Subroutine Tij_READ}

This subroutine reads the unique hopping matrix elements and their connectivities from the input file. In case the system consists of more than one unit cell, the routine also generates rest of the hoppings from translational invariance.

\subsection{Subroutine Tij GEN}

This routine is used in order to generate hopping automatically from the information of bond distance and the corresponding hopping matrix elements. The subroutine considers all pairs of sites in the molecule and if the distance between them is equal to the user provided distance, it assigns to them the

user provided hopping matrix elements. This automates the generation of hopping matrix elements to a great extent, thus, simplifying the input data. This routine is invoked by the card 'HOPGEN' in the input file.

\subsection{Subroutine Vij_CAL}

This subroutine computes the long-range Coulomb matrix elements $V_{i j}$ (cf. Eqs. 2, 3, \& 4) for the P-P-P model with three possible parametrization namely Ohno, Mataga-Nishimoto, or exponential, depending upon the input provided. In case the choice of Hamiltonian is the Hubbard or the extended Hubbard model, the relevant Coulomb parameters $U$ and/or $V$, are provided in the input itself. In case of RHF calculations, the routine can also calculate the contribution corresponding to the correlation functions, if such a calculation has been requested in the input file. For any choice of a correlated Hamiltonian, corresponding interaction matrix elements are stored in an array. 


\subsection{Subroutine BANDS}

This is the subroutine which performs the band structure calculations using the Hückel model. In order to get the band structure, first Fourier transformation of the hopping matrix elements is performed, and later the matrix is diagonalized using the subroutine ZHPEV from the LAPACK/BLAS library to obtain the eigenvalues and eigenvectors. The eigenvalues of corresponding bands are written in an ASCII file 'bands.dat' which can be used for plotting the bands using standard programs such as xmgrace[34] or gnuplot[35]. The eigenvectors are written in the binary format, in the file named 'bloch_orbitals.dat'.

\subsection{Subroutine FOUTRA_TB}

This subroutine computes the Fourier transform of one dimensional nearestneighbor-interacting tight-binding Hamiltonian. It is called by subroutine BANDS, discussed above.

\subsection{Subroutine $S C F_{-} R H F$}

This subroutine solves the RHF equations for the system under consideration in a self-consistent manner, using the iterative diagonalization procedure and returns the canonical SCF orbitals, their eigenvalues, and the total energies. The arrays which are needed during the calculations are allocated before the calculations begins, and are deallocated upon completion. Before the first iteration, Hückel model Hamiltonian is diagonalized to obtain a set of starting orbitals. Subsequently, the Fock matrix corresponding to those orbitals is constructed and diagonalized. The process is repeated until the self-consistency is achieved. During the self-consistency iterations, subroutine DSPEVX from the LAPACK/BLAS library is used to obtain the occupied eigenvalues and eigenvectors. Obtaining only the occupied eigenpairs, as against the entire spectrum, leads to considerable savings of CPU time for large systems. However, if the entire spectrum of eigenvalues and eigenvectors is needed, say, to perform optical absorption calculations or preparing the data for subsequent correlation calculations, the Fock matrix is diagonalized using the routine DSPEV from the LAPACK/BLAS library, upon completion of the self-consistency iterations. Because the entire spectrum is obtained only once the self-consistency has been achieved, it does not strain the computational resources too much. 


\subsection{Subroutine $S C F+U H F$}

This subroutine is exactly the same in its logic and structure as the previously described SCF_RHF, except that the task of this routine is to solve the UHF equation for the system under consideration. Different Fock matrices for the up- and the down-spin are constructed and diagonalized in each iteration, until the self-consistency is achieved. Similar to the case of routine SCF_RHF, during the iterations only the occupied eigenvalues and eigenvectors are computed using the routine DSPEVX. The iterations are stopped once the total UHF energy of the system converges to within a user defined threshold.

\subsection{Subroutine DENSITY}

This subroutine constructs the density matrix for the closed-shell systems, assuming orbitals to be doubly occupied.

\subsection{Subroutine DENSITY UHF}

This subroutine constructs density matrices needed for the UHF calculations. It generates different density matrices for the orbitals with up $(\alpha)$ and down $(\beta)$ spins, and in the end adds them to compute the total density matrix.

\subsection{Subroutine FOCKMAT}

This subroutine constructs the Fock matrix for the closed-shell system and calculates the total SCF energy from the density matrix and the one- and two-electron integrals.

\subsection{Subroutine FOCKMAT_UHF}

This subroutine is analogous to the routine FOCKMAT, the only difference is that it constructs the Fock matrix for the UHF calculations by computing separate Fock matrices for up- and down-spins. Thereafter, it calculates the total SCF energy from the density matrices and the one- and two-electron integrals. 


\subsection{Subroutine ORBDEN}

This routine computes the charge density of a given set of orbitals on user specified sites. This helps in understanding as to how the charge is distributed in an extended system and helps us to visualize that whether a given orbital is localized or delocalized. This analysis can be performed both on RHF and UHF orbitals.

\subsection{Subroutine WRITORB}

The purpose of this subroutine is to write the converged orbitals to the orbital file in the ASCII format. In case of RHF calculations, orbitals will be written in 'ORB001.DAT' file, while in case of UHF calculations, orbitals with upand down-spins will be written in the files 'ORBALPHA.DAT' and 'ORBBETA.DAT', respectively.

\subsection{Subroutine SCI}

This subroutine is the master routine for performing the single configuration interactions (SCI) calculations, using the Hartree Fock occupied and virtual MOs. SCI is a simple approach which accounts for the electron-correlation effects by including configurations which are singly-excited with respect to the HF reference state, in the CI expansion[2]. Because of the Brillouin theorem, which forbids the mixing of the HF state with the singly-excited configurations, the ground state remains unchanged in a SCI calculation[2]. However, excited configurations do mix with each other leading to a reasonably good description of the excited states in this approach[2]. In the present version of code, SCI method is implemented only for the case of closed-shell systems, for which RHF calculations are performed prior to the SCI ones. To obtain the CI eigenvalues, SCI matrix is diagonalized using the routine DSPEV from the LAPACK/BLAS library.

\subsection{Subroutine HAM_SCI}

This routine constructs the single-CI Hamiltonian matrix for the singlet subspace. A closed-shell reference state is assumed. The Hamiltonian matrix elements between different sets of configurations are computed using the standard Slater-Condon rules[2]. 


\subsection{Subroutine OPTICS}

This is the master routine meant for computing the optical absorption spectrum, which can be obtained at the single particle level (TB or HF) as well as at the SCI level. The range of frequencies over which the spectrum is to be computed, along with the line width, are read from the input file. The calculated spectrum is written in an output file called 'spec001.dat', which can be plotted using xmgrace[34] or gnuplot[35]. At present this routine is restricted to the closed-shell systems.

\subsection{Subroutine SPCTRM_A}

This routine computes the absorption spectrum from the ground state under electric-dipole approximation, assuming a Lorentzian line shape and a constant line width for all the levels. The computed spectrum is written in an ASCII file 'spec001.dat', which can be readily used for plotting using programs such as xmgrace[34] or gnuplot[35].

\subsection{Subroutine SPCTRM_1EX}

This is an important subroutine which calculates the linear optical absorption of the system from a singly-excited state (as compared to the ground state) for one electron TB or HF calculation. The formalism as well as the approach of computation is same as that in routine SPCTRM_A, and the output of this routine is also written in the ASCII file 'spec001.dat'. Excited state absorption spectra are useful in interpreting photo-induced absorption experimental data.

\subsection{Subroutine DIPMAT}

If optical absorption calculations are to be performed within the SCI approach, one needs electric dipole matrix elements between various singly excited configurations. The task of this routine is to compute these matrix elements so that they can be used for calculating the optical absorption spectrum in the routine SPCTRM_A. 


\subsection{Subroutine NLO}

This subroutine generates the input file to be used for the nonlinear optical (NLO) susceptibility calculations at the single particle level. In order to reduce the size of dipole matrix elements, one can delete the outermost occupied and virtual orbitals choosing the card 'ORBDEL'. The program deletes the orbitals in the given range using a subroutine named 'delo_nlo', which will be described next. Output data of NLO calculations is written in file 'NLO001.DAT', which includes total number of orbitals, number of occupied orbitals, energies of the occupied, as well as virtual states, and the dipole moments. NLO option is also restricted to the RHF calculations only. This data can be used to compute NLO susceptibilities such as two-photon absorption (TPA), third harmonic generation (THG), etc., using the sum-over-states (SOS) formalism of Orr and Ward[36].

\subsection{Subroutine DELO_NLO}

The task of this subroutine is to delete occupied and virtual orbitals from the orbital array for the NLO calculations. This routine is called by subroutine NLO, if needed.

\subsection{Subroutine DIPCAL}

This routine computes the dipole moment matrix between the single particle orbitals under the Hückel approximation, according to which, among the AOs only diagonal matrix elements are nonzero, and their values are nothing but their Cartesian locations. This routine simply transforms the dipole matrix elements from the AO representation to the MO one. This routine is called from the routine NLO and its aim is to supply dipole integrals needed for subsequent SOS calculations of NLO susceptibilities mentioned above.

\subsection{Subroutine $C I \_D R V$}

This is the driver routine which controls the preparations of various data files needed for the post-HF correlated calculations, such as FCI, QCI, MRSDCI, etc. The tasks of this routine include:

(1) Reading the orbitals

(2) Freezing and/or deleting orbitals if specified by the user in the input file 
(3) Creating the one-electron effective core potential (ECP) corresponding to the frozen set of orbitals, if needed

(4) Transforming the one- and two-electron integrals from the site (AO) representation to the basis spanned by these orbitals (MOs). They are written to different files so that they can be read and used by a separate post-HF correlation program.

\subsection{Subroutine ECP}

This program calculates the effective one-electron frozen core potential matrix elements, and stores them in the array. Eventually, these matrix elements are added to the other one-electron part of the Hamiltonian before being written out in the CI_DRV output files. The energy contribution of the frozen orbitals is stored in the variable ecore.

\subsection{Subroutine TWOIND}

This routine performs the two-index transformation on one-electron matrix elements, so as to transform them from the AO to the MO representation for the use in subsequent correlation calculations. It is called by the master routine CI_DRV.

\subsection{Subroutine WRITE_1}

This routine writes all the nonzero one-electron Hamiltonian matrix elements expressed in the MO representation in a binary file called 'ONEINT001.DAT'. This routine is also called by the master routine CI_DRV, and the output is meant to be used in the post-HF correlation calculations.

\subsection{Subroutine FOURIND}

This routine performs the four-index transformation on the two-electron integrals so as to transform them from the AO to the MO represention. This routine is also called by the master routine CI_DRV. 


\subsection{Subroutine WRITE_2}

This routine writes all the nonzero two-electron Hamiltonian matrix elements expressed in the MO representation in a binary file called 'TWOINT001.DAT'. This routine is also called by the master routine CI_DRV, and the output is meant to be used in the post-HF correlation calculations.

\subsection{Subroutine DIPINT}

This routine transforms the dipole operator from the site (AO) representation into the representation of the orbitals (MOs). The routine is called from CI_DRV and it provides dipole matrix elements to be used for optical properties calculations in conjunction with post-HF correlated calculations. The algorithm used in this routine is the one described in the routine DIPCAL.

\subsection{Subroutine DIPOUT}

The purpose of this routine is to write all the nonzero dipole matrix elements in the binary file 'DIPINT001.DAT' to be used in conjunction with the post-HF correlated calculations. This routine is also called from CI_DRV.

\section{Installation, input files, output files}

We believe that the installation and execution of the program, as well as preparation of suitable input files is fairly straightforward. Therefore, we will not discuss these topics in detail here. Instead, we refer the reader to the README file for details related to the installation and execution of the program. Additionally, the file 'manual.pdf' explains how to prepare a sample input file. Several sample input and output files corresponding to various example runs are also provided with the package.

\section{$5 \quad$ Results and Discussions}

In this section, we present and discuss the numerical applications of our code. First we present the results on the convergence of total energy with respect to the oligomer's length for various conjugated polymers such as $t$-PA, PPP, 
PPV, and polyacene. We also provide our results for the UHF calculation on graphene nanodisks. Next, we present our results for the tight binding band structure calculations for PPP. Further, we discuss and compare the linear absorption optical spectrum of PPP computed by different approaches such as TB, HF, and SCI. As mentioned in the above sections that the program can perform calculations in the presence of finite electric field as well, which makes it capable of calculating electro-absorption spectrum. For centro-symmetric systems, the electro-absorption spectrum allows one to explore both the even and the odd parity excited states in a linear optical absorption process. Results and analysis of the electro-absorption spectrum of an oligomer of PPP at the SCI level are also presented.

\subsection{Total Energy}

Our code can be used to study both the ground and the excited state properties of oligomers of various polymers because they are nothing but finite molecules, ranging in size from small to large. However, in this section we demonstrate that our code can also be used to obtain the ground state energy/cell, in the bulk limit, for one-dimensional periodic systems such as polymers.

The energy per unit cell of a one-dimensional periodic system can be obtained using the formula

$$
E_{\text {cell }}=\lim _{n \rightarrow \infty} \Delta E_{n}=\lim _{n \rightarrow \infty}\left(E_{n+1}-E_{n}\right),
$$

where $E_{n+1} / E_{n}$ represent the total energies of oligomers containing $n+1 / n$ unit cells. Thus, using this formula, for sufficiently large value of $n$, one can obtain the energy/cell of a polymer in the bulk limit, from oligomer based calculations. In what follows we show that value of $E_{\text {cell }}$ converges quite rapidly with respect to $n$.

First we examine the convergence of $E_{\text {cell }}$ obtained using Eq. (13) with respect to the number of unit cells $n$, using the RHF approach. The energy convergence threshold for the SCF calculations is set up to eighth decimal place. In Fig. 1 we plot $\Delta E_{n}$ as a function of $n$, for various polymers such as $t$-PA, acene, PPP, and PPV. From plots (c) and (d) in Fig. 1, it is evident that energy for PPP and PPV converges rapidly and convergence is achieved for short oligomer length (6-7 units), while in t-PA and acene (Fig. 1(a) and (b), respectively), the convergence is achieved for larger value of $n$.

The above mentioned calculations can also be performed using the UHF approach. However, it is more meaningful to use this approach for systems with open-shell configurations, such as trigonal zigzag graphene-nanodisks. Thus, UHF calculations can be used to ascertain whether or not a high-spin state is 


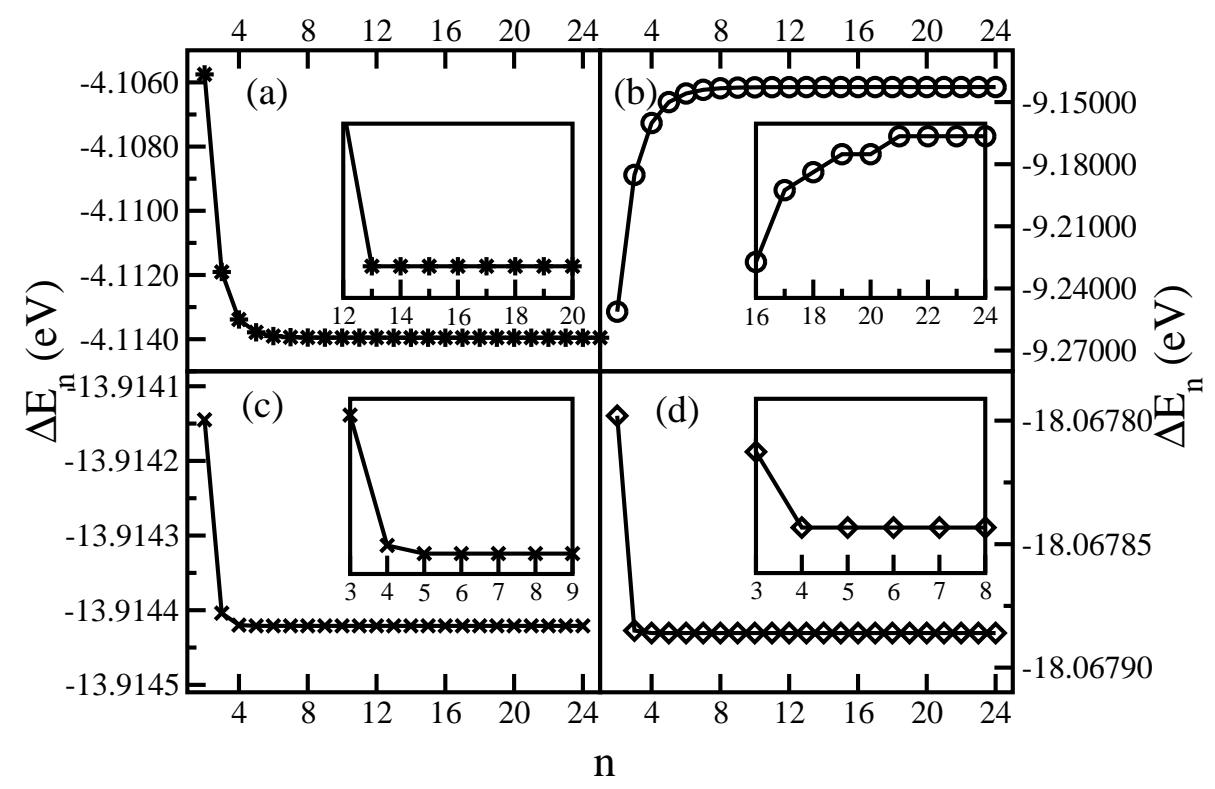

Figure 1. Convergence in $\triangle E_{n}$ with respect to the oligomer length $(n)$ of various systems: (a) $t$-PA, (b) polyacene, (c) PPP, and (d) PPV. Inset of each graph shows the zoomed plot, in the energy window of $10^{-7}-10^{-8} \mathrm{eV}$.

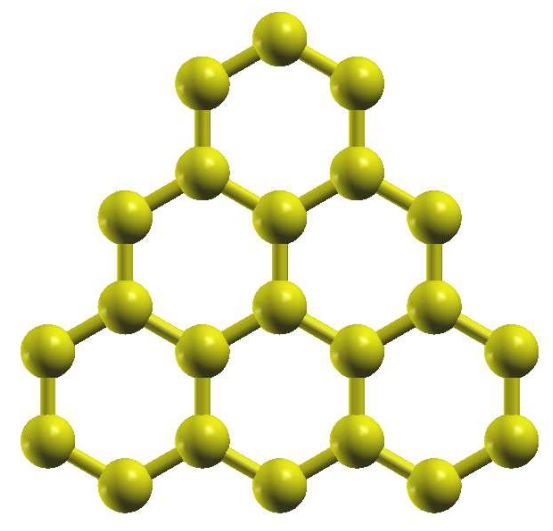

Figure 2. Schematic diagram of the trigonal zigzag graphene nanodisk with six benzene rings (22 sites) considered in this work.

the ground state. For example, we found that the ground state of a trigonal zigzag graphene-nanodisk with six benzene rings ( $c f$. Fig. 2) is a triplet one (see Table 1).

\subsection{Band Structure}

Our code can be used to perform band structure calculations using the nearestneighbor TB model. In Fig. 3, we present band structure of PPP. It possesses six bands out of which two are localized (labeled as ' $l$ ', ' $l^{*}$ ), while four are 
Table 1

Results of UHF total energy calculations on a trigonal zigzag graphene nanodisk with six benzene rings ( $c f$. Fig. 2) for various combinations of majority $\left(n_{\alpha}\right)$ and minority $\left(n_{\beta}\right)$ spins. Our results indicate that the triplet state being significantly lower in the total energy than the singlet state, is the true ground state.

\begin{tabular}{|c|c|c|}
\hline$n_{\alpha}$ & $n_{\beta}$ & $E(\mathrm{eV})$ \\
\hline \hline 11 & 11 & -49.840415300708 \\
\hline 12 & 10 & -51.858572334959 \\
\hline 13 & 9 & -48.083423107736 \\
\hline 14 & 8 & -43.974251795664 \\
\hline
\end{tabular}

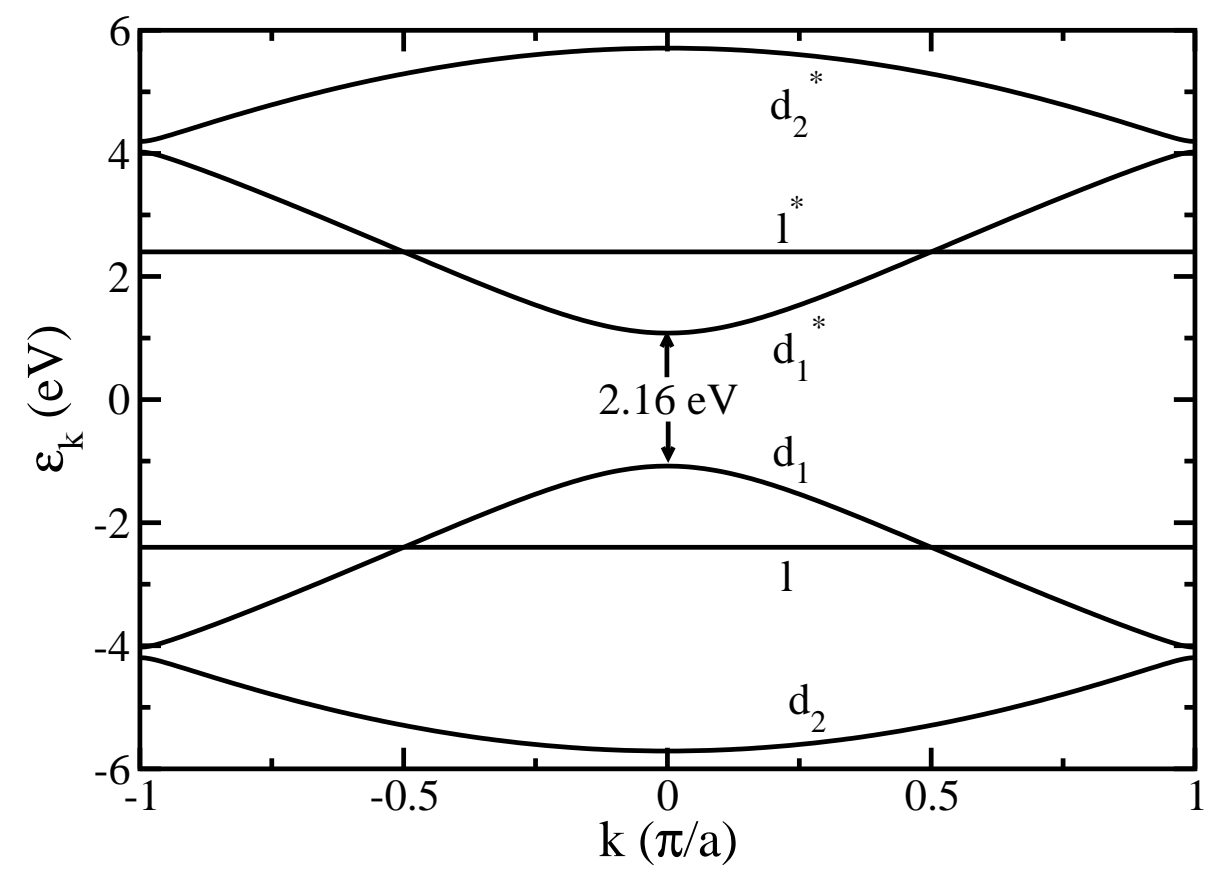

Figure 3. Band structure of PPP, computed using Hückel model Hamiltonian. Dispersion less flat bands denote the localized levels $\left(l / l^{*}\right)$, while the other bands represent the delocalized ones.

delocalized (labeled as ' $d_{1} / d_{2}$ ', ' $d_{1}^{*} / d_{2}^{*}$ ). As is obvious from the Fig. 3 , the band structure has a direct band gap, $E_{g}=2.16 \mathrm{eV}$ at the $\Gamma$-point. Our calculated band structure is fully consistent with that reported by Heeger and co-workers[37]. 


\subsection{Linear optical absorption spectrum}

In order to understand the optical properties of conjugated polymers, it is essential to study their low-lying excited states. The linear optical absorption spectrum, which is all about one-photon absorption, provides an efficient medium to explore the odd-parity low-lying states in centro-symmetric systems. In an independent particle model it can be explained in terms of excitations from ground state, by promoting one electron from one of the occupied MO (valence band states) to one of the unoccupied MO (conduction band states). While, in a correlated electron approach, such as the P-P-P model, this one-electron picture does not hold, and the excited states are linear combination of several configurations obtained by performing CI calculations.

Our code can be used to compute linear optical absorption spectrum of $\pi$ conjugated materials using the Hückel model (TB approach), as well as P-P-P model Hamiltonians employing both the HF and the SCI approaches.

In Fig. 4 we present the linear optical absorption spectrum of planar PPP with eight repeat units (PPP-8) using TB, HF, and SCI approaches. The spectrum was calculated using the carbon-carbon bond length (within a phenyl ring) to be $1.4 \AA$, while the inter-ring single bond length was taken to be $1.54 \AA$. The standard value of $-2.4 \mathrm{eV}$ was used for the intra-benzene hopping, while for the inter-ring single bond we used the value $-2.23 \mathrm{eV}$. The $\mathrm{HF}$ and the SCI calculations were performed using the standard parameters in the P-P-P Hamiltonian. All the three graphs depicted in Fig. 4 are qualitatively quite similar, with the first two features, and the most intense feature, corresponding to the $x$-polarized transition. The spectrum drawn at the HF level using P-P-P model is blue shifted as compared to the Hückel model spectrum, which is due to the well known tendency of the HF approach to over estimate the band gaps. However, inclusion of correlation effects at the SCI level brings the spectrum back to lower energy range. The location of the lowest peak corresponds to the optical gap, which is found to be around $3.5 \mathrm{eV}$, in excellent agreement with the reported first-principles calculations[38], and also in good agreement with the experimental value $(\approx 3 \mathrm{eV})$ for the infinite polymer[39]. A higher level CI calculations like FCI, QCI, and MRSDCI can also be performed using, e.g., the MELDF code[40] beyond HF. The input for these higher CI calculations can be prepared using CIPREP option in the input file.

On analyzing the wave functions of the excited states contributing to the spectra, we find that first peak in $\mathrm{TB}$ and $\mathrm{HF}$ spectra is due to transition from the highest occupied molecular orbital (HOMO) to the lowest unoccupied molecular orbital (LUMO), i.e., $H \rightarrow L$. In the spectrum obtained using SCI approach, for peak I (see Fig. 4), the major contribution comes from $H \rightarrow L$ configuration, while the contributions due to $H-1 \rightarrow L+1, H-2 \rightarrow L+2$, 


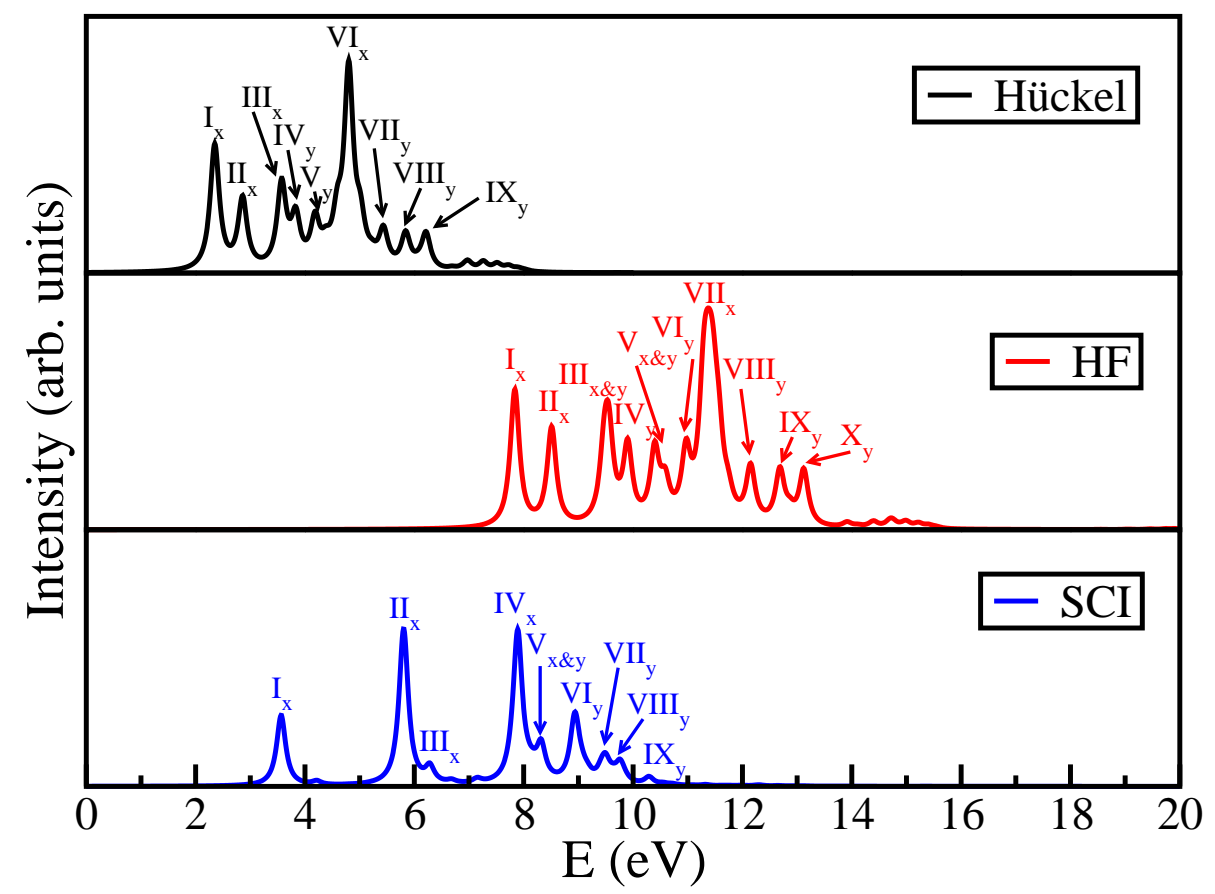

Figure 4. Linear optical absorption spectrum of planar PPP-8, computed using the Hückel as well as P-P-P models, both at the HF and the SCI levels. Various peaks and their polarizations are labeled. The spectra are plotted using the line width of $0.1 \mathrm{eV}$.

$H-3 \rightarrow L+3$, and $H-12 \rightarrow L+12$ are also significant.

Our code is also capable of examining the role of Coulomb parameters used in the $\mathrm{P}-\mathrm{P}-\mathrm{P}$ Hamiltonian by not only considering standard and screened set of parameters defined earlier ( $c f$. Sec. 2), but also any user defined set of Coulomb parameters, which may be material specific. Fig. 5 depicts the linear absorption spectra computed using standard parameters (dashed line) and screened parameters (solid line), for planar geometry of PPP-8 at SCI level. Both the spectra are qualitatively similar, while quantitatively, spectrum computed using screened parameters is red shifted as compared to the standard parameter spectrum. We note that the value $3.27 \mathrm{eV}$ of the optical gap obtained using the screened parameters is in better agreement with the experimental value $(\approx 3 \mathrm{eV})$ for the infinite system[39].

\subsection{Electro-absorption spectrum}

As mentioned earlier our code can also solve the HF equations in the presence of a finite electric field. This allows us, in principle, to use it for computing quantities such as the static dielectric polarizabilities and the electroabsorption (EA) spectra of various conjugated systems. For example, the firstorder polarizability is determined as the second derivative of the total energy 


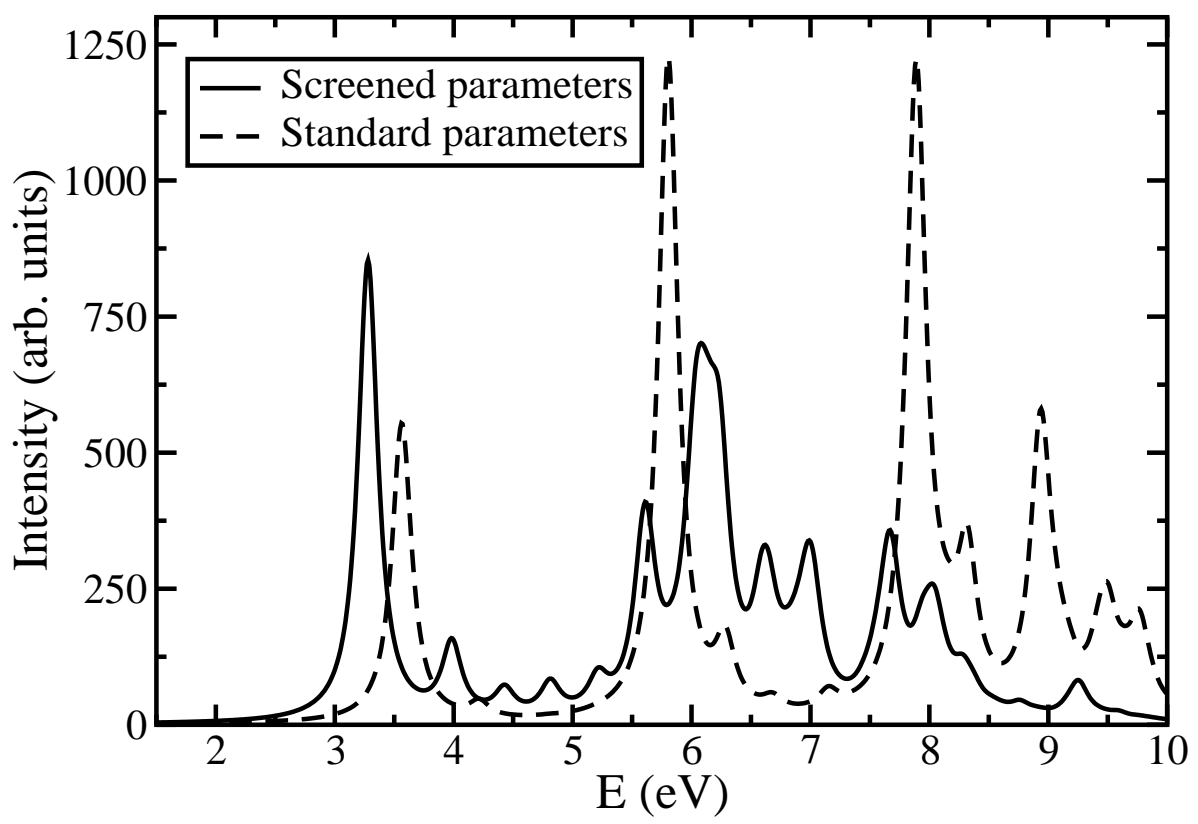

Figure 5. Linear optical absorption spectrum of PPP-8 computed using the standard (dashed line) as well as the screened (solid line) set of Coulomb parameters in the P-P-P model, using the SCI approach. The spectra are plotted using the line width of $0.1 \mathrm{eV}$.

per unit cell $\left(E_{\text {total }}\right)$ with respect to the strength of the external electric field $\mathbf{E}$. The second derivative of $E_{\text {total }}$ can be calculated employing finite difference formulas, using $E_{\text {total }}$ with, and without, the electric field. However, in this work we are concentrating on another possible application of the finite-field approach, namely the electro-absorption spectrum.

The electro-absorption spectrum is an important experimental probe for various materials which, through linear optical absorption allows, one to probe both even, as well as odd parity states for centro-symmetric systems. The noteworthy point is that without electro-absorption normally one will have to use both linear absorption as well as nonlinear absorption to investigate states of both parities. Using our program one can easily make theoretical prediction of this quantity using its definition: $\Delta \alpha(\omega, \mathbf{E})=\alpha(\omega, \mathbf{E})-\alpha(\omega, 0)$, where $\alpha(\omega, \mathbf{E})$ is the optical absorption spectrum in the presence of the external electric field E. In order to compute it, the HF equations are solved in the presence of the field $\mathbf{E}$ ( $c f$. Eq. 6) leading to a modified set of orbitals and their eigenvalues, as compared to the zero-field $(\mathbf{E}=0)$ case. This orbital set can subsequently be used to compute: (a) optical absorption at the HF level, or (b) optical absorption at any level of correlation. Then by using the above mentioned definition of $\Delta \alpha(\omega, \mathbf{E})$ one can compute the corresponding EA spectrum. In this work we present the results of the EA spectrum of PPP-8, computed at the SCI level using our program. The EA spectra are usually interpreted in 

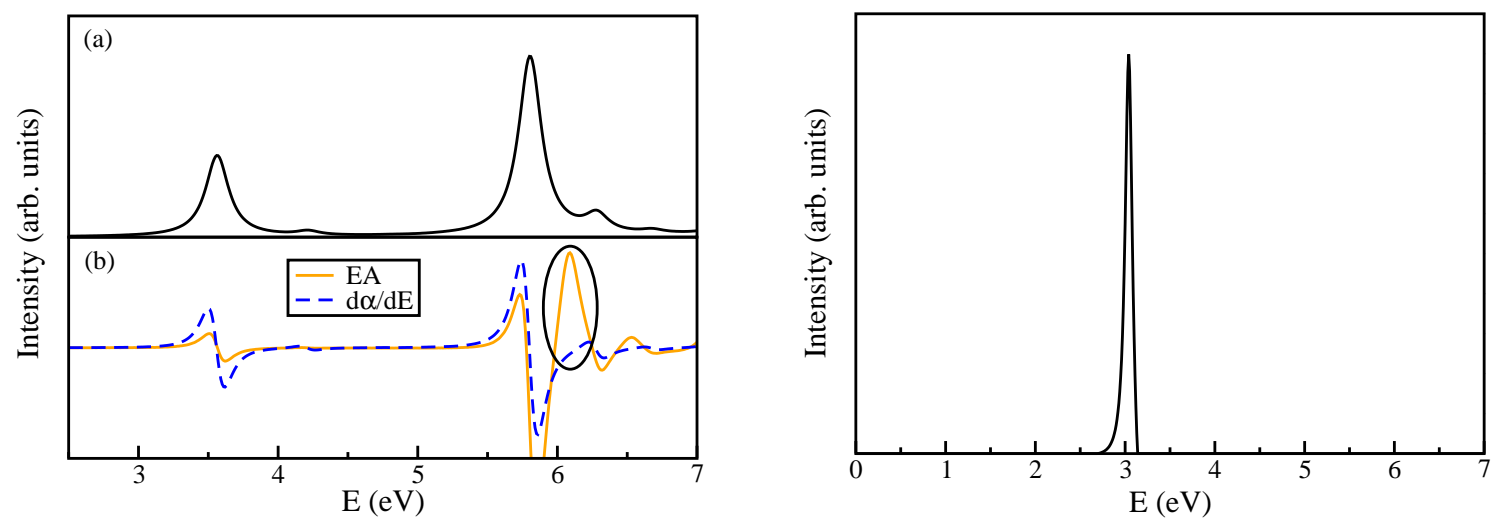

Figure 6. Left panel: (a) The linear absorption spectrum planar molecule of PPP-8, computed in the presence of finite electric field, using standard parameters in the P-P-P Hamiltonian with the SCI approach. (b) Electro-absorption spectrum (solid line) and the first derivative of linear absorption with respect to the photon energy $\mathrm{E}$ (dashed line) for the same system. Right panel: The two photon absorption (TPA) spectrum of PPP-8, computed at SCI level, using the MELDF program[40]. In all the figures above, E represents the photon energy. The $m A_{g}$ state in the TPA spectrum corresponds to the encircled feature in the EA spectrum, as is also clear by the peak positions.

terms of Stark shift analysis and it roughly follows the first derivative of linear absorption, calculated in the presence of the electric field, with respect to the photon energy.

Fig. 6 (a) shows the linear absorption spectrum computed in the presence of finite electric field, using the standard parameters in the P-P-P Hamiltonian with the SCI approach. As expected, the most intense features in the EA spectrum appears when the field is applied along the conjugation direction which happens to be the $x$-axis in the present case. In these calculations the electric field strength was taken to be $10^{-3} \mathrm{~V} / \AA$, which is of the same order of magnitude used in various experiments[41]. Fig. 6 (b) depicts the EA spectrum together with the first derivative of linear absorption with respect to the photon energy E. We note that the EA spectrum resembles the first derivative of linear absorption spectrum, roughly. The first absorption peak in linear absorption spectrum occurs at $3.56 \mathrm{eV}$, following the other features at $4.21 \mathrm{eV}, 5.8 \mathrm{eV}$, and $6.27 \mathrm{eV}$ [see Fig. 6 (a)]. In EA spectrum, the first peak appears at $3.51 \mathrm{eV}$, which is similar to $\mathrm{d} \alpha / \mathrm{dE}$ spectrum [dashed curve in Fig. 6 (b)]. We therefore assign this feature to a Stark shift of the lowest odd parity $\left(1 B_{u}\right)$ exciton. There is also a feature at $6.09 \mathrm{eV}$ [circled in Fig. 6 (b)], which cannot be found by a derivative analysis of the absorption spectrum, and must therefore be due to an even parity $\left(m A_{g}\right)$ exciton. To verify this, we also calculated two photon absorption (TPA) spectrum using the MELDF code[40] at the SCI level. The right panel of Fig. 6 shows our calculated TPA spectrum for PPP-8. The spectrum features an intense peak located at 3.04 $\mathrm{eV}$, which exhibits strong dipole coupling with the lowest one-photon $1 B_{u}$ 
state, and therefore is the $m A_{g}$ state[42], located at $6.08 \mathrm{eV}$. Thus, our result for $m A_{g}$ state obtained from the EA spectrum is in excellent agreement with that from the TPA.

\section{Conclusions and Future Directions}

In this paper we have described our Fortran 90 program which solves the HF equations for both the closed- and open-shell molecular systems using the semi-empirical Hückel and PPP models. To demonstrate the features of our code, we presented results of numerous test calculations on various molecular systems. The reason behind developing the present program is twofold: (a) to develop a code in a modern language such as Fortran 90 which can carry out dynamic array allocation, and thus, free the user from specifying and changing array sizes, and (b) to provide an open software which will be widely available to users which they can use and modify as per their needs. One possible generalization of this code will be to include the capabilities for calculations on infinite systems in one- and higher dimensions to calculate the band structure and related properties using the P-P-P model. Work along those directions is continuing in our group, and results will be published as and when they become available.

\section{Acknowledgements}

Authors thank the Department of Science and Technology (DST), Government of India, for providing financial support for this work under Grant No. SR/S2/CMP-13/2006.

\section{References}

[1] W. Barford, Electronic and Optical Properties of Conjugated Polymers (Clarendon Press, Oxford, 2005).

[2] See, e.g., L. Salem, Molecular orbital theory of conjugated systems, W. A. Benjamin Inc., New York (1966).

[3] D. Fichou, ed., Handbook of Oligo- and Polythiophenes (Wiley-VCH, Wienheim, 1999); M . A. Baldo, M. E. Thompson, and S. R. Forrest, Nature (London) 403 (2000) 750; S. R. Forrest, Nature (London) 428 (2004) 911; G. Malliaras and R. Friend, Phys. Today 58 (2005) 53; H. Sirringhaus, N. Tessler, and R. H. Friend, Science 280 (1998) 1741. 
[4] A. K. Geim and Novoselov, Nature Materials 6 (2007) 183.

[5] A. H. Castro Neto, F. Guinea, N. M. R. Peres, K. S. Novoselov, and A. K. Geim, Rev. Mod. Phys. 81 (2009) 109.

[6] R. Pariser and R. G. Parr, J. Chem. Phys. 21 (1953) 767; J. A. Pople, Trans Faraday Soc. 49 (1953) 1275.

[7] See, e.g., D. Barieswyl, D. K. Campbell, and S. Mazumdar, in Conjugated Conducting Polymers, edited by H. Keiss (Springer-Verlag, Berlin, 1992), pp. $7-133$.

[8] See, e.g., K. Jug, Int. J. Quant. Chem. 37 (1990) 403.

[9] Z. G. Soos, S. Ramasesha, and D. S. Galvão, Phys. Rev. Lett. 71 (1993) 1609.

[10] A. Ye, Z. Shuai, and J. L. Brédas, Phys. Rev. B 65 (2002) 045208.

[11] C. Raghu, Y. Anusooya Pati, and S. Ramasesha, Phys. Rev. B 66 (2002) 035116.

[12] R. J. Bursill and W. Barford, J. Chem. Phys. 130 (2009) 234302.

[13] D. Psiachos and S. Mazumdar, Phys. Rev. B 79 (2009) 155106.

[14] A. Shukla and S. Mazumdar, Phys. Rev. Lett 83 (1999) 3944.

[15] H. Ghosh, A. Shukla, and S. Mazumdar, Phys. Rev. B 62 (2000) 12763.

[16] A. Shukla, H. Ghosh, and S. Mazumdar, Synth. Met. 116 (2001) 87.

[17] A. Shukla, Chem. Phys. 300 (2004) 177.

[18] A. Shukla, Phys. Rev. B 69 (2004) 165218.

[19] P. Sony and A. Shukla, Phys. Rev. B 71 (2005) 165204.

[20] A. Shukla and P. Sony, Synth. Met. 155 (2005) 368.

[21] A. Shukla, Phys. Rev. B 65 (2002) 125204.

[22] A. Shukla, H. Ghosh, and S. Mazumdar, Phys. Rev. B 67 (2003) 245203.

[23] A. Shukla, H. Ghosh, and S. Mazumdar, Synth. Met. 141 (2004) 59.

[24] P. Sony and A. Shukla, Synth. Met. 155 (2005) 316.

[25] P. Sony and A. Shukla, Phys. Rev. B 71 (2005) 165204.

[26] P. Sony and A. Shukla, J. Chem. Phys. 131 (2009) 014302.

[27] A. Shukla (unpublished).

[28] K. Ohno, Theor. Chim. Acta 2 (1964) 219.

[29] N. Mataga and K. Nishimoto, Z. Physik. Chemie 12 (1957) 140.

[30] K. Schulten, I. Ohmine, and M. Karplus, J. Chem. Phys. 64 (1976) 4422. 
[31] M. Chandross and S. Mazumdar, Phys. Rev. B 55 (1997) 1497.

[32] K. Dharamvir and V. K. Jindal (unpublished).

[33] A. Kokalj, Comp. Mat. Sci. 28 (2003) 155.

[34] Xmgrace is a two-dimensional plotting tool for $\mathrm{X}$ Window based systems available at URL http://plasma-gate.weizmann.ac.il/Grace.

[35] Gnuplot is a public domain command-line driven interactive data and function plotting utility available at URL http://www.gnuplot.info.

[36] B.J. Orr and J.F. Ward, Mol. Phys. 20 (1971) 513.

[37] E. K. Miller, G. S. Maskel, C. Y. Yang, and A. J. Heeger, Phys. Rev. B 60 (1999) 8028.

[38] C. Ambrosch-Draxl, J. A. Majewski, P. Vogl, G. Leising, R. Abt, and K. D. Aichholzer, Synth. Met. 69 (1995) 411.

[39] L. Athouël, J. Wéry, B. Dulieu, J. Bullot, J. P. Buisson, and G. Froyer, Synth. Met. 84 (1997) 287.

[40] MELDF, a molecular electronic structure program from University of Indiana with contributions from E. R. Davidson, L. McMurchie, S. Elbert, and S. Langhoff. Program No: 580, Quantum Chemistry Program Exchange, Indiana University, Bloomington, Indiana, USA.

[41] P. A. Lane, M. Liess, Z. V. Vardeny, M. Hamaguchi, M. Ozaki, and K. Yoshino, Synth. Met. 84 (1997) 641.

[42] S.N. Dixit, D. Guo, and S. Mazumdar, Phys. Rev. B 43 (1991) 6781; S. Mazumdar and F. Guo, J. Chem. Phys. 100 (1994) 1665. 